\title{
Biased random walks and propagation failure
}

\author{
Vicenç Méndez, ${ }^{1}$ Sergei Fedotov, ${ }^{2}$ Daniel Campos, ${ }^{1}$ and Werner Horsthemke ${ }^{3}$ \\ ${ }^{1}$ Grup de Física Estadística, Departament de Física, Facultat de Ciències. Edicifi Cc, Universitat Autónoma de Barcelona, 08193 \\ Bellaterra, Barcelona, Spain \\ ${ }^{2}$ School of Mathematics, The University of Manchester, M60 1QD, United Kingdom \\ ${ }^{3}$ Department of Chemistry, Southern Methodist University, Dallas, Texas, 75275-0314, USA
}

(Received 3 October 2006; revised manuscript received 4 January 2007; published 19 January 2007)

\begin{abstract}
The critical value of the reaction rate able to sustain the propagation of an invasive front is obtained for general non-Markovian biased random walks with reactions. From the Hamilton-Jacobi equation corresponding to the mean field equation we find that the critical reaction rate depends only on the mean waiting time and on the statistical properties of the jump length probability distribution function and is always underestimated by the diffusion approximation. If the reaction rate is larger than the jump frequency, invasion always succeeds, even in the case of maximal bias. Numerical simulations support our analytical predictions.
\end{abstract}

DOI: 10.1103/PhysRevE.75.011118

Random walk models are frequently used to describe the movement and dispersal of animals or microorganisms, see, e.g., Ref. [1] and references therein. Arguably, the simplest random walk is Brownian motion. The random walk is biased if jumps in one particular direction are more likely and a drift in that direction will be observed [2]. Biased random walks have been used to account for foraging performance in heterogeneous landscapes [3], for sensory, physiological, and cognitive responses in micro-organisms [4], and for kinesis and taxis [5]. They have also been considered in other fields of science, e.g., in studies of imperfections in the source of randomness [6], faster file location in $\mathrm{P} 2 \mathrm{P}$ networks [7], in percolation models for myopic ants [8], particle diffusion in disordered solids [9], and in disorderly fractal structures [10].

In many applications, transport processes occur together with reaction processes. In the case that dispersal is simple Brownian motion, the mean-field evolution equation of the system is a reaction-diffusion equation. Such equations have been used in physics, chemistry, and biology to model a wide variety of phenomena, including the invasion of a linearly unstable state by a propagating front $[1,11,12]$. An interesting question arises when the dispersal is biased in a direction away from the region occupied by the unstable state. What are the conditions on the reaction rate and bias that will result in a stalled front? Or phrased differently, what is the critical (minimal) value of the reaction rate to sustain front propagation when the underlying random walk has a bias in the opposite direction. Our main results are as follows: (i) The standard diffusion approximation of the transport process always provides an inaccurate value for the critical reaction rate. (ii) If the reaction rate exceeds the jump frequency of the random walk, then the front cannot stall and will always propagate into the unstable state, independently of the values of the other statistical parameters of the random walk.

We choose the initial conditions to be $n(0, x)=1$ for $x \leqslant 0$ and $n(0, x)=0$ for $x>0$, where $n(t, x)$ is the density of particles at $x$ at time $t$. This initial condition describes, for example, a territory divided into an invaded zone $(x \leqslant 0)$ and a noninvaded zone $(x>0)$, separated by a frontier (at $x=0)$. For the reaction process, we choose Fisher-Kolmogorov-
Petrovski-Piskunov (FKPP) kinetics [13], the standard form in studies of traveling fronts. If particles or individuals disperse according to an isotropic random walk with FKPP kinetics, the above initial condition turns into a front propagating from left to right, i.e., the invasion starts. Our choice of initial conditions ensures that the propagation speed of the front is minimal [14]. Since the particle jumps are isotropic, the reaction is responsible for the motion of the front from left to right. It is the reaction process that starts and maintains a successful invasion. A bias to the left in the random walk will hinder the invasion. Therefore we expect that the critical reaction rate is given by a balance between the factor favoring the invasion (reaction process) and the factor opposing the invasion (bias in the transport process).

Our goal is to derive an analytical expression for the critical reaction rate in terms of the joint probability density function (pdf) $\Phi(t, x)$ for the waiting times $t$ and jumps $x$ of the underlying random walk. The mean field equation for $n(t, x)$ can be written in the form [15]

$$
\begin{aligned}
n(t, x)= & \Psi(t) n(0, x)+\int_{0}^{t} \int_{-\infty}^{\infty} \Phi(s, z) n(t-s, x-z) d z d s \\
& +U \int_{0}^{t} \Psi(s) n(t-s, x)[1-n(t-s, x)] d s,
\end{aligned}
$$

where $\Psi(t)$ is the probability that particles do not move up to time $t$ and $\psi(t)$ is the waiting time pdf,

$$
\Psi(t)=1-\int_{0}^{t} \psi(s) d s, \quad \psi(t)=\int_{-\infty}^{\infty} \Phi(t, x) d x .
$$

The standard diffusion approximation of (1), i.e., taking the limit of small jump lengths and small waiting times, yields the reaction-diffusion-advection equation $\partial_{t} n-c \partial_{x} n=D \partial_{x x} n$ $+U n(1-n) \quad$ where $c=\langle x\rangle /\langle t\rangle, D=\left\langle x^{2}\right\rangle / 2\langle t\rangle,\langle t\rangle=\int_{0}^{\infty} t \psi(t) d t$, and $\left\langle x^{j}\right\rangle=\int_{-\infty}^{\infty} x^{j} \rho(x) d x$ are the moments of the dispersal kernel $\rho(x)=\int_{0}^{\infty} d t \Phi(x, t)$.

We use the Hamilton-Jacobi approach to determine the propagation speed of the front. To do this, we make use of the hyperbolic scaling $x \rightarrow x / \varepsilon, t \rightarrow t / \varepsilon$ and represent the 
rescaled density $n^{\varepsilon}(x, t)=n(x / \varepsilon, t / \varepsilon)$ in the form $n^{\varepsilon}(x, t)=\exp \left[-G^{\varepsilon}(x, t) / \varepsilon\right]$ with $G^{\varepsilon}(x, t) \geqslant 0$. As long as $G(x, t)=\lim _{\varepsilon \rightarrow 0} G^{\varepsilon}(x, t)>0$ then $n^{\varepsilon}(x, t) \rightarrow 0$ as $\varepsilon \rightarrow 0$. The boundary of the set where $G(x, t)>0$ can be regarded as the front position. Substitution of the rescaled density into (1) and taking the limit $\varepsilon \rightarrow 0$ one finds that the Hamilton-Jacobi equation for (1) is given by [15]

$$
1-\hat{\Phi}(H, p)-\frac{U}{H}[1-\hat{\psi}(H)]=0 .
$$

The moment generating functions $\hat{\Phi}(H, p)$ and $\hat{\psi}(H)$ are defined as

$$
\begin{gathered}
\hat{\Phi}(H, p)=\int_{0}^{\infty} d s \int_{-\infty}^{\infty} \Phi(s, z) e^{-H s} e^{p z} d z \\
\hat{\psi}(H)=\int_{0}^{\infty} \psi(s) e^{-H s} d s .
\end{gathered}
$$

The propagation speed $u$ is obtained from two equations involving $H$ and $p$ [16],

$$
u=\frac{H}{p}, \quad \frac{\partial H}{\partial p}=\frac{H}{p} .
$$

The critical condition, i.e., a stalled front, $u=0$, is realized if $H\left(p^{*}\right)=0$ with $p^{*}>0$. Writing the Laplace transform $\hat{\psi}(H)$ as a power series of $H, \hat{\psi}(H)=1-\langle t\rangle H+\cdots$, and taking the limit $H \rightarrow 0$ in (3), we obtain $1-\hat{\Phi}(0, p)-\langle t\rangle U^{*}=0$. Differentiating (3) and taking into account that $u=0$ in (6), we find that the critical value of the momentum $p$ is the solution of $d \hat{\Phi}(0, p) / d p=0$. This results in the following simple form for the critical reaction rate:

$$
U^{*}=\frac{1-\hat{\rho}\left(p^{*}\right)}{\langle t\rangle},
$$

with $p^{*}$ such that

$$
\left.\frac{d \hat{\rho}(p)}{d p}\right|_{p=p^{*}}=0,
$$

where $\hat{\rho}(p)=\int_{-\infty}^{\infty} e^{p x} \rho(x) d x$. Note that the critical value $U^{*}$ depends on the shape of dispersal kernel and on the mean waiting time $\langle t\rangle$ only. This implies that two systems with identical dispersal kernels, different waiting times pdfs, but the same mean waiting time $\langle t\rangle$, will have the same $U^{*}$. Since $\hat{\rho}(p)>0$, the maximum value for $U^{*}$ is $1 /\langle t\rangle$. When $U=U^{*}$, front propagation fails. The front speed is 0 due to the opposing effects of the reaction and jump processes. If $U>U^{*}$, the front moves from left to right. When $U<U^{*}$, $u<0$ and the front moves from right to left. An interesting way to interpret Eq. (7) is as a "balance" or an "equilibrium" condition between parameters favorable (left-hand side) and unfavorable (right-hand side) to the invasion. However, there is another way to look at this relation. The product $\langle t\rangle U$ is a dimensionless quantity, the reaction-diffusion number. Equation (7) implies that $U>U^{*}$ is equivalent to
$N_{\mathrm{GRD}} \equiv\langle t\rangle U+\hat{\rho}\left(p^{*}\right)>1$, where we have defined the generalized reaction-diffusion number $N_{\mathrm{GRD}}$. If $N_{\mathrm{GRD}}>1$, the front travels from left to right $(u>0)$. If $N_{\mathrm{GRD}}<1$, the front travels from right to left, and if $N_{\mathrm{GRD}}=1$ the front stalls and turns into a stationary spatial pattern $(u=0)$. If $U$ is larger than the maximum possible value of $U^{*}$, then $u>0$ and invasion always succeeds no matter how large the opposing bias in the transport process. From (7), max $U^{*}=1 /\langle t\rangle$, and we conclude that if $U>1 /\langle t\rangle$, i.e., the reaction rate is larger than the jump frequency, the front always propagates to the right, completely independent of the other statistical properties of the random walk.

We now prove that the diffusion approximation always underestimates $U^{*}\left(U_{\mathrm{dif}}^{*}<U^{*}\right)$. To do so, it is sufficient to consider a small bias to the left such that

$$
\int_{-\infty}^{0} \rho(x) d x-\int_{0}^{\infty} \rho(x) d x \equiv \boldsymbol{\epsilon}
$$

and $0<\epsilon \ll 1$. The normalization condition implies

$$
\int_{-\infty}^{0} \rho(x) d x+\int_{0}^{\infty} \rho(x) d x=1 .
$$

First, we determine the order of magnitude of the moments of $\rho(x)$. For $j=1,2, \ldots$ one can write $\left\langle x^{j}\right\rangle$ $=\lim _{\ell \rightarrow \infty}\left[\int_{-\ell}^{0} x^{j} \rho(x) d x+\int_{0}^{\ell} x^{j} \rho(x) d x\right]=\lim _{\ell \rightarrow \infty} \int_{0}^{\ell} x^{j}\left[\rho(x)+(-1)^{j}\right.$ $\times \rho(-x)] d x$. Using the mean value theorem for integrals and (9), we find

$$
\begin{aligned}
\left\langle x^{2 j}\right\rangle & =\lim _{\ell \rightarrow \infty} \int_{0}^{\ell} x^{2 j}[\rho(x)+\rho(-x)] d x \\
& =\xi_{1}^{2 j} \lim _{\ell \rightarrow \infty} \int_{0}^{\ell}[\rho(x)+\rho(-x)] d x=\xi_{1}^{2 j} \sim O(1)
\end{aligned}
$$

and

$$
\begin{aligned}
\left\langle x^{2 j-1}\right\rangle & =\lim _{\ell \rightarrow \infty} \int_{0}^{\ell} x^{2 j-1}[\rho(x)-\rho(-x)] d x \\
& =\xi_{2}^{2 j} \lim _{\ell \rightarrow \infty} \int_{0}^{\ell}[\rho(x)-\rho(-x)] d x=-\xi_{2}^{2 j-1} \epsilon \sim O(\epsilon),
\end{aligned}
$$

for some $\xi_{1}$ and $\xi_{2}$ in $[0, \ell]$. The minus sign in (11) implies $\left\langle x^{2 j-1}\right\rangle<0$ and reflects that the random walk is biased to the left. As a result we can write

$$
\hat{\rho}(p)=\sum_{j=0}^{\infty} \frac{\left\langle x^{j}\right\rangle p^{j}}{j !}
$$

We break off the expansion at order $j=3$, i.e., a small correction to the diffusion approximation (order $j=2$ ):

$$
\hat{\rho}(p)=1+p\langle x\rangle+\frac{1}{2} p^{2}\left\langle x^{2}\right\rangle+\frac{1}{6} p^{3}\left\langle x^{3}\right\rangle
$$

and find 

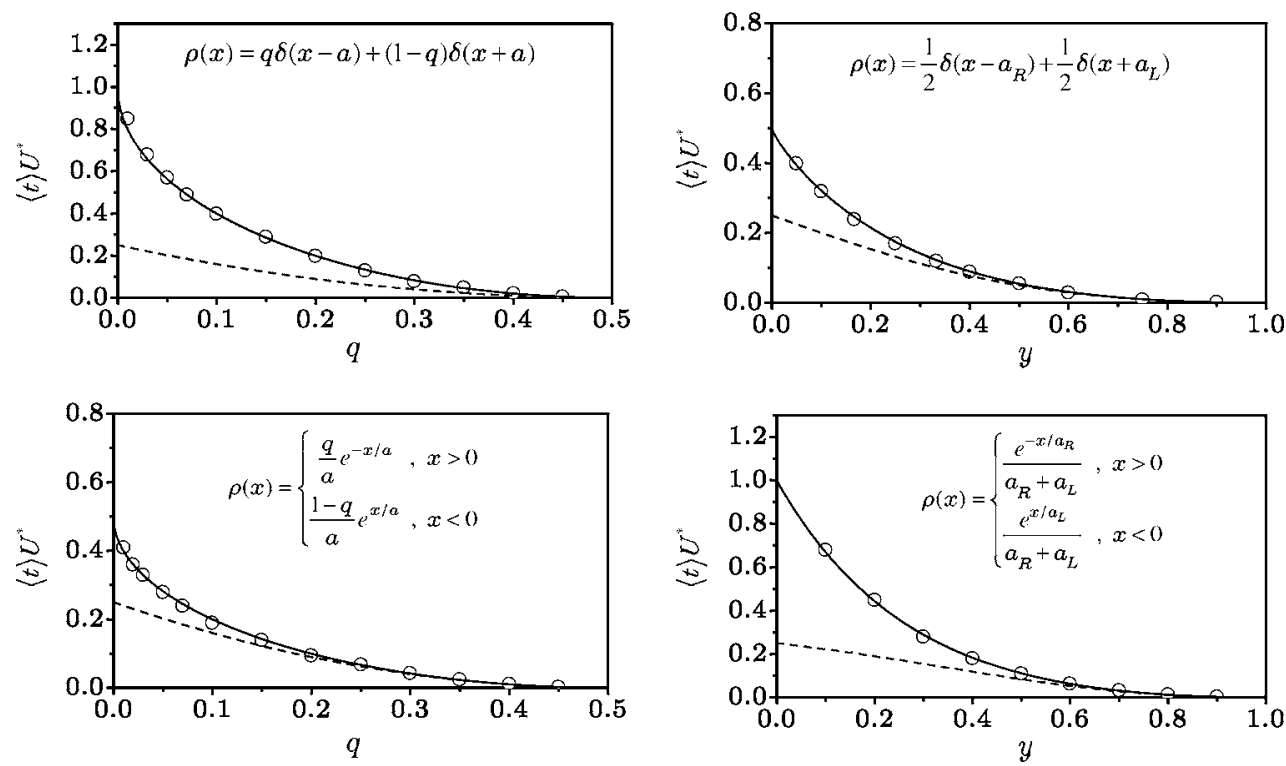

FIG. 1. The dimensionless critical reaction rate, calculated analytically (solid lines), obtained numerically (open circles), and using the diffusion approximation (dashed lines), is plotted versus the dimensionless parameters $q$ or $y$.

$$
\begin{aligned}
p^{*} & =\frac{\left\langle x^{2}\right\rangle}{\left\langle x^{3}\right\rangle}\left(-1+\sqrt{1-2 \frac{\langle x\rangle\left\langle x^{3}\right\rangle}{\left\langle x^{2}\right\rangle^{2}}}\right) \\
& \simeq-\frac{\langle x\rangle}{\left\langle x^{2}\right\rangle}\left(1+\frac{1}{2} \frac{\langle x\rangle\left\langle x^{3}\right\rangle}{\left\langle x^{2}\right\rangle^{2}}\right)+O\left(\epsilon^{4}\right),
\end{aligned}
$$

where we have made use of (10) and (11) to compute the series expansion. Inserting (13) into (12) and expanding according to (10) and (11), we obtain

$$
\hat{\rho}\left(p^{*}\right)=1-\frac{1}{2} \frac{\langle x\rangle^{2}}{\left\langle x^{2}\right\rangle}-\frac{1}{6} \frac{\langle x\rangle^{3}\left\langle x^{3}\right\rangle}{\left\langle x^{2}\right\rangle^{3}} .
$$

As the condition for the critical reaction rate is $\langle t\rangle U^{*}=1-\hat{\rho}\left(p^{*}\right)$, one has for the diffusion approximation

$$
\langle t\rangle U_{\mathrm{dif}}^{*}=\frac{1}{2} \frac{\langle x\rangle^{2}}{\left\langle x^{2}\right\rangle},
$$

and finally

$$
\langle t\rangle U^{*}-\langle t\rangle U_{\mathrm{dif}}^{*}=\frac{1}{6} \frac{\langle x\rangle^{3}\left\langle x^{3}\right\rangle}{\left\langle x^{2}\right\rangle^{3}}>0 .
$$

The last inequality results from the fact that the odd moments are negative. In conclusion, $U^{*}>U_{\text {dif }}^{*}$, i.e., the diffusion approach always underestimates the critical reaction rate.

We illustrate our theoretical results by considering a simple dispersal kernel $\rho(x)$ with an effective drift from right to left. There are two simple ways to introduce such a drift: (i) the probability of a jump to the left is greater than the probability of the same jump to the right or (ii) jumps to the right and left have the same probability but are of different length. Consider first a Diracdelta kernel, i.e., a dispersal kernel with fix jump length, $\rho(x)=q \delta(x-a)+(1-q) \delta(x+a), 0<q<1 / 2$. Using (7) and (14), we obtain

$$
\begin{aligned}
& \langle t\rangle U^{*}=1-2 \sqrt{q(1-q)}, \quad\langle t\rangle U_{\mathrm{dif}}^{*}=\frac{1}{2}(1-2 q)^{2} . \\
& \text { If } \rho(x)=\frac{1}{2} \delta\left(x-a_{R}\right)+\frac{1}{2} \delta\left(x-a_{L}\right), \text { then } \\
& \langle t\rangle U^{*}=1-\frac{1}{2}\left(y^{-y /(1+y)}+y^{1 /(1+y)}\right), \quad\langle t\rangle U_{\mathrm{dif}}^{*}=\frac{(1-y)^{2}}{4\left(1+y^{2}\right)},
\end{aligned}
$$

where $0<y=a_{R} / a_{L}<1$. Next we consider an exponential distribution with different statistical characteristics for $x \geqslant 0$ and $x<0$. Let $\rho(x)$ be $q e^{-x / a} / a$ for $x \geqslant 0$ and $(1-q) e^{x / a} / a$ for $x<0$. Then,

$$
\langle t\rangle U^{*}=1-\frac{(1-2 q)^{2}}{2-4 \sqrt{q(1-q)}}, \quad\langle t\rangle U_{\mathrm{dif}}^{*}=\frac{1}{4}(1-2 q)^{2} .
$$

If $\rho(x)$ is $e^{-x / a_{R}} /\left(a_{R}+a_{L}\right)$ for $x \geqslant 0$ and $e^{x / a_{L}} /\left(a_{R}+a_{L}\right)$ for $x<0$ then,

$$
\langle t\rangle U^{*}=\left(\frac{1-y}{1+y}\right)^{2}, \quad\langle t\rangle U_{\mathrm{dif}}^{*}=\frac{(1-y)^{2}}{4\left(1-y+y^{2}\right)} .
$$

To check our analytical results, we have performed simulations that mimic the dispersal-reaction processes microscopically and have found, for every biased form of $\rho(x)$ considered above, the critical value $U^{*}$ at which wave propagation stalls. In our simulations [17], we consider a 1D lattice where dispersal and reaction processes take place starting from steplike initial conditions. Instead of studying the evolution of a large number of individual particles, as is usually done, we consider a continuous density of particles $n(t, x)$ for every cell in the lattice. For the case $\rho(x)=q \delta(x-a)+(1-q) \delta(x+a)$ dispersal works in this way: a fraction $q$ of the density $n(t, x)$ in a certain cell goes to the cell at $x+a$ and a fraction $1-q$ goes to the cell at $x-a$ [for nondiscrete forms of $\rho(x)$ we discretize the corresponding 
function]. After that, the reaction function is applied to each cell in the lattice. We also take the time between successive steps in this scheme equal to $\langle t\rangle$. The computation time required is significantly reduced in comparison with methods based on the evolution of individual particles. However, the rules imply that our simulations are deterministic, and they do not account for effects due to the discreteness of particles, such as cutoff effects [18]. In consequence, we stress that our method is really appropriate for comparison with mesoscopic models as those presented here. A more extensive study of this issue has been conducted recently by two of the authors [17]. Figure 1 shows that the numerical simulations (open circles) are in excellent agreement with the analytical predictions (solid curves) given in (16)-(19) for Dirac-delta and exponential kernels. Moreover, the dashed curves correspond to the diffusion approximation and lie below the solid curves, in agreement with the general result given in Eq. (15).

In summary, we have employed reaction-continuous-time random walks as a mesoscopic framework to study the opposing effects of biased random walks and logistic reactions on front propagation. From the Hamilton-Jacobi equation for the mean field equation we have found a relation that allows us to compute analytically the critical reaction rate $U^{*}$. A main result is that $U^{*}$ depends on the dispersal kernel and on the mean waiting time only, i.e., $U^{*}$ does not depend on the shape of the waiting-time pdf. In particular, we have shown that if the reaction rate is larger than the jump frequency, the invasion front never fails, independent of the shape of the waiting-time pdf, even if the dispersal kernel is maximally biased. A second important result is that the diffusion approximation always underestimates $U^{*}$. Numerical simulations show excellent agreement with the analytical results. Propagating fronts are observed in numerous fields. Concrete examples are fronts in solid fuel combustion [19], reaction fronts in polymerization systems [20], front propagation in chemical systems with applied electric fields [21,22], chemotactic waves in amoeba [23], spread of populations through river networks [24], invasion of foreign species [25], spread of diseases such as rabies [26] or foot-and-mouth disease [27], fronts in cardiac tissue [28], and the invasion of gliomas, a highly malignant brain tumor $[29,30]$. Conditions that result in a stalled front are of crucial importance in these applications.

This work has been partially funded by Grants Nos. FIS 2006-12296-C02-01 and SGR 2005-00087 (V.M.), EPSRC EP/D03115X/1 (V.M. and S.F.) and by the National Science Foundation under Grant No. CHE-0533642 (W.H.).
[1] J. D. Murray, Mathematical Biology (Springer-Verlag, Berlin, 1989).

[2] H. C. Berg, Random Walks in Biology (Princeton University Press, Princeton, NJ, 1983).

[3] D. Grünbaum, Am. Nat. 151, 97 (1998).

[4] N. A. Hill and D. P. Häder, J. Theor. Biol. 186, 503 (1997).

[5] E. A. Codling and N. A. Hill, J. Theor. Biol. 233, 573 (2005).

[6] M. Santha and U. Vazirani, J. Comput. Syst. Sci. 33, 75 (1986)

[7] Y. Azar, A. Z. Broder, A. R. Karlin, N. Linial, and S. Phillips, 16, 18 (1996).

[8] R. B. Pandey, D. Stauffer, A. Margolina, and J. G. Zabolitsky, J. Stat. Phys. 34, 427 (1986).

[9] J. P. Bouchaud and A. Georges, Phys. Rep. 125, 127 (1990); E. Arapaki, P. Argyrakis, I. Avramov, and A. Milchev, Phys. Rev. E 56, R29 (1997).

[10] P. Meakin, Phys. Rev. B 28, 5221 (1983).

[11] M. C. Cross and P. C. Hohenberg, Rev. Mod. Phys. 65, 851 (1993).

[12] Oscillations and Traveling Waves in Chemical Systems, edited by R. J. Field and M. Burger (Wiley, New York, 1985).

[13] R. A. Fisher, Proc. Annu. Symp. Eugen. Soc. 7, 355 (1937); A. N. Kolmogorov, I. G. Petrovskii, and N. S. Piskunov, Moscow University Mathematics Bulletin 1, 1 (1937).

[14] D. G. Aronson and H. F. Weinberger, Adv. Math. 30, 33 (1978)
[15] S. Fedotov and V. Méndez, Phys. Rev. E 66, 030102(R) (2002).

[16] S. Fedotov, Phys. Rev. Lett. 86, 926 (2001).

[17] D. Campos, V. Méndez, and V. Ortega-Cejas (unpublished).

[18] E. Brunet and B. Derrida, Phys. Rev. E 56, 2597 (1997).

[19] B. J. Matkowsky and G. I. Sivashinsky, SIAM J. Appl. Math. 35, 465 (1978).

[20] M. Apostolo, A. Tredici, M. Morbidelli, and A. Varma, J. Polym. Sci., Part A: Polym. Chem. 35, 1047 (1997).

[21] E. P. Zemskov, V. S. Zykov, K. Kassner, and S. C. Müller, Physica D 183, 117 (2003).

[22] Z. Virányi, D. Horváth, and A. Tóth, J. Phys. Chem. A 110, 3614 (2006).

[23] R. E. Goldstein, Phys. Rev. Lett. 77, 775 (1996).

[24] D. Campos, J. Fort, and V. Méndez, Theor Popul. Biol. 69, 88 (2006).

[25] A. Okubo, P. K. Maini, M. H. Williamson, and J. D. Murray, Proc. R. Soc. London 238, 113 (1989).

[26] J. D. Murray, E. A. Stanley, and D. L. Brown, Proc. R. Soc. London 229, 111 (1986).

[27] R. P. Pech and J. C. McIlroy, J. Appl. Ecol. 27, 635 (1990).

[28] V. N. Biktashev, Phys. Rev. Lett. 89, 168102 (2002).

[29] K. R. Swanson, C. Bridge, J. D. Murray, and E. C. Alvord, Jr., J. Neurol. Sci. 216, 1 (2003).

[30] E. Khain and L. M. Sander, Phys. Rev. Lett. 96, 188103 (2006). 